\title{
BANCO DE SEMILLAS DEL SUELO EN UN ÁREA DE BOSQUES NATIVOS SUJETA A CAMBIO EN EL USO DE LA TIERRA (ENTRE RÍOS, ARGENTINA)
}

\author{
SIONE, S. M. J. ${ }^{1} ;$ LedesmA, S. G. ${ }^{1} ;$ ROSEnBERGER, L. J. ${ }^{1}$; \\ Wilson, M. G. ${ }^{1,2} \&$ SABATtini, R. A. ${ }^{2}$
}

\begin{abstract}
RESUMEN
El objetivo es analizar el banco de semillas del suelo (BSS) en un área de bosques nativos sujeta a cambio de uso de la tierra, y evaluar su rol en la regeneración de estos ecosistemas. Se evaluaron cuatro tratamientos: bosque nativo $(\mathrm{BN})$, bosque nativo con manejo ganadero (BNM), bosque en regeneración (BR) y agricultura (AGR). La mayor densidad del BSS (11.917 semillas. $\mathrm{m}^{-2}$ ) se registró en BNM, correspondiendo a AGR el menor valor (3.142 semillas. $\left.\mathrm{m}^{-2}\right)$. Vachellia caven resultó la única especie arbórea representada en el banco, con densidades relativas inferiores al 1\%. Las especies invasoras dominaron en todos los tratamientos, a excepción del BNM, que presentó mayor contribución de Gramíneas forrajeras. E1 85\% de las malezas determinadas en AGR fueron registradas en los otros tratamientos. El alto grado de fragmentación de los bosques nativos de la Cuenca, asociado al avance de la frontera agrícola, facilitaría el flujo de semillas de especies invasoras desde áreas agrícolas hacia los bosques circundantes, constituyendo otro de los impactos negativos de la deforestación.

Palabras claves: agroecosistemas boscosos, regeneración, reservorio de propágulos, sucesión vegetal.
\end{abstract}

\section{ABSTRACT}

\section{Soil seed bank in an area of native forests associated with change in land use (Entre Rios, Argentina).}

The aim is to analyze the soil seed bank (SSB) in an area of native forests subject to change in land use and assess their role in natural regeneration of these ecosystems. Four treatments were evaluated: Native forest (NF), native forest in livestock management (NFM), regeneration forest (RF) and agriculture (AGR). The seed total density was significantly higher in NFM (11917seeds. $\left.\mathrm{m}^{-2}\right)$ than other treatments, corresponding to AGR the lowest value $\left(3142\right.$ seeds. $\left.\mathrm{m}^{-2}\right)$. Vachellia caven was the only tree species found, with values of relative density less than $1 \%$ in the forests evaluated. Weed species constituted the largest group of percentage contribution to SSB in all treat-

1.- Facultad de Ciencias Agropecuarias. Universidad Nacional de Entre Ríos. Ruta 11 - Km. 10.5 (3101) Oro Verde, provincia de Entre Ríos.

2.- INTA EEA Paraná. Ruta 11 - Km. 12.5 (3101) Oro Verde, provincia de Entre Ríos.

Manuscrito recibido el 4 de febrero de 2016 y aceptado para su publicación el 24 de mayo de 2016. 
ments, except NFM, dominated by Gramineae forage. The $85 \%$ of the weeds identified in AGR were found in the other treatments. The high degree of fragmentation of native forests, associated with the advance of the agricultural frontier, facilitate the seed flow of invasive species from agricultural areas into nearby forests, constituting one of the negative impacts of deforestation.

Key words: forest agroecosystems, regeneration, propagules stock, vegetal succession.

\section{INTRODUCCIÓN}

La República Argentina ha experimentado históricamente un intenso proceso de transformación de sus bosques nativos, debido al avance de la frontera agrícola y al aprovechamiento forestal selectivo. Se estima que desde 1935 se ha perdido cerca del $70 \%$ de los bosques (40), con una tasa actual de deforestación de 250000 ha por año (49). La provincia de Entre Ríos no es ajena a esta situación forestal, observándose una drástica reducción, fragmentación y degradación estructural y funcional de los bosques, como consecuencia de la deforestación para la reconversión a tierras agrícolas, la sobreexplotación forestal selectiva $y$ la ganadería extensiva con pastoreos no planificados. Estas actividades han generado cambios en las formaciones leñosas, con predominio de bosques secundarios degradados, invasión de especies leñosas exóticas y empobrecimiento de pastizales naturales (38).

Cuando las áreas incorporadas a la agricultura a partir de la deforestación son abandonadas, se desencadenan procesos de sucesión vegetal que propician el establecimiento de nuevas comunidades vegetales, diferentes a las prístinas. La regeneración natural es determinante en la conservación de un sistema y su estudio es básico para entender el reemplazo de especies en una comunidad (50). El banco de semillas del suelo (BSS), entendido como el almacén 2 de semillas viables que potencialmente son capaces de germinar y establecerse (7), representa el potencial regenerativo de las comunidades vegetales y cumple un papel preponderante en la recuperación de áreas disturbadas (11, 19). Luzuriaga et al. (25) sostienen que las semillas y propágulos almacenados en el suelo, juegan un papel crucial en la colonización de hábitats perturbados, en aquellos casos en que la lluvia de semillas presenta una contribución limitada.

Los BSS son muy dinámicos y presentan alta heterogeneidad en composición y abundancia, tanto espacial como temporal (30), siendo afectados por la frecuencia e intensidad de los disturbios (27). En este sentido, la composición del BSS brinda información acerca del estado de la sucesión vegetal, de las especies potencialmente capaces de reemplazar a las existentes y del tipo de especies que componen la vegetación presente y circundante $(3,39)$. Altas densidades de especies malezas en el BSS pueden desviar o detener la sucesión del bosque secundario (12), constituyendo fuentes de invasión de especies no nativas en lugar de una fuente potencial de regeneración (23).

Thompson et al. (46) indican que una especie puede formar un banco transitorio (Bt) o persistente (BP). Las semillas que conforman el Bt presentan una viabilidad inferior a un año, mientras que los BP presentan semillas con mayor viabilidad (44). Los bancos persistentes revisten mayor importancia con relación a la regeneración (15).

| Revista FAVE - Ciencias Agrarias 15 (1) 2016 